\title{
Gastric Melanoma of Unknown Primary
}

\author{
Fabio Cortellini ${ }^{1,2}$, Giovanni Marasco ${ }^{1,2}$, Matteo Renzulli $^{1}$, Francesco Vasuri ${ }^{1}$, Luigi Ricciardiello ${ }^{1,2}$
}

1) Azienda Ospedaliero-Universitaria di Bologna, Bologna; 2) Department of Medical and Surgical Sciences, University of Bologna, Bologna, Italy
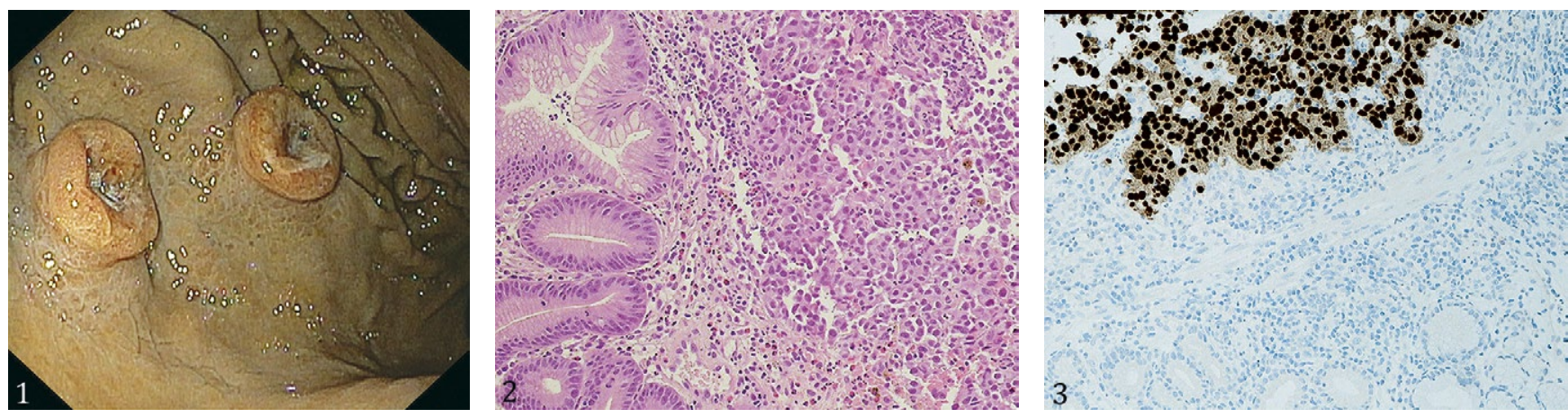

An 81-year-old male patient was admitted to the Geriatric ward for progressive weakness, hyporexia and anemia. The physical examination revealed epigastric abdominal pain triggered by palpation. No history of gastro-intestinal hemorrhage or constipation was reported. Laboratory tests showed severe microcytic anemia (hemoglobin: $7.4 \mathrm{~g} / \mathrm{dl}$ ) and slight c-reactive protein elevation $(1.39 \mathrm{mg} / \mathrm{dl})$. The patient was referred to our Endoscopy Unit and underwent an upper digestive endoscopy, which identified three vegetant lesions in the greater curvature of the gastric body (Fig. 1). The lesions were polypoid, measuring up to $15-20 \mathrm{~mm}$ in diameter, and presenting an ulcerated central depression, aberrant mucosal pattern, and with soft consistency when biopsied. The histopathological examination showed epithelioid neoplastic infiltration of the gastric mucosa (Fig. 2), with immunochemistry positivity at SOX-10 and s100, consistent with gastric melanoma metastasis (Fig. 3).

A thorough dermathologic inspection with epiluminescence, and an examination of fundus oculi were made, which failed to find the primary lesion. Hence, the final diagnosis was metastatic melanoma with unknown primary.

Melanoma of unknown primary is defined as a metastatic melanoma within the lymph nodes, subcutaneous tissues, and other distant sites without an evident primary lesion [1], and its incidence is low (3.2\%) among all the cases of melanoma [2]. The occurrence of gastric metastasis is around $0.2-0.7 \%$ in endoscopic and autoptic studies, and melanoma is the most common primary tumor [3]. Melanoma and breast cancer have an organotropism for the gastro-intestinal tract, and this is probably the reason why they are responsible for the majority of gastrointestinal metastasis [2]. Gastric metastasis may present with various morphologies, often indistinguishable from primary neoplastic lesions. Therefore, endoscopic biopsy is crucial to characterise these lesions [4] and sometimes represents the sole key step capable of guiding clinicians in the diagnostic process, as highlited by this case.

Corresponding author: Giovanni Marasco, giovannimarasco89@gmail.com

Conflicts of interest: None to declare.

Acknowledgments: We thank Alberto Bagni MD for his contribution to the histologic examination of the case.

\section{REFERENCES}

1. Song Y, Karakousis GC. Melanoma of unknown primary. J Surg Oncol 2019;119:232-241. doi:10.1002/jso.25302

2. Kamposioras K, Pentheroudakis G, Pectasides D, Pavlidis N. Malignant melanoma of unknown primary site. To make the long story short. A systematic review of the literature. Crit Rev Oncol Hematol 2011;78:112-126. doi:10.1016/j.critrevonc.2010.04.007

3. Haendchen Bento L, Kazuyoshi Minata M, Pires Batista C, et al. () Clinical and endoscopic aspects of metastases to the gastrointestinal tract. Endoscopy 2019;51:646-652. doi:10.1055/a-0887-4401

4. Kim GH, Ahn JY, Jung HY, et al. Clinical and endoscopic features of metastatic tumors in the stomach. Gut Liver 2015;9:615-622. doi:10.5009/gnl14032 